\title{
MEDICINE AND MATERIALS
}

Volume 1, Issue 1, 2021: 31-42 | ISSN: 2784-1499 \& e-ISSN: 2784- 1537

DOI: 10.36868/MEDMATER.2021.01.01.031 | www.medicineandmaterials.com |

\section{BIOTERRORISM AND BIOSECURITY - RISK ANALYSIS AND SPECIFIC PREVENTION}

\author{
Madalina Maria DIAC, Nona GIRLESCU*, Iuliana HUNEA, \\ Simona Irina DAMIAN, Anton KNIELING, Diana BULGARU ILIESCU \\ Institute of Legal Medicine, University of Medicine and Pharmacy "Grigore T Popa” Iasi, Romania
}

\begin{abstract}
Microorganisms with lethal potential for human body and the toxins they produce have their own place in history, because of the numerous epidemics they have caused and because of the large number of human lives they have taken. They are a hidden enemy, extremely insidious and with high mortality rates and they can destroy all the defence, control, and medical systems of a state; they can kill many people before anyone can realise the true phenomenon; they can hide the criminals before even suspecting the crime, and they can create chaos, terror, and even economic collapse. Furthermore, we have to analyse the conceptual mutations of the terrorist phenomenon in the last decade. It tends to be a mass destruction act rather than a manipulation and ideologic movement. We can truly say that bioterrorism is the scariest scenario of all existing threats and the main question remains: are all international systems ready to face this enemy and is there a prevention protocol that could be adapted realistically to these threats?
\end{abstract}

Keywords: biological weapon, terrorism, biowarfare, epidemics, biosafety, biological prevention

\section{Introduction}

The terrorist phenomenon has evolved massively in the past few years and - besides the extent and realism of this danger - whatever its nature: religious, ethnical, etc., the interest in mass destruction weapons represents a warning sign in what concerns international security. In the hierarchy of mass destruction weapons, the most disastrous scenario is the one-off using highrisk biological agents, which can produce dreadful epidemics, hard to control and to treat; besides the massive human loss, it may cause economic damage able to destabilize a state, maybe even irremediably [1].

The Centre for Disease Control and Prevention in the US has ranked these microorganisms into three classes of risk, by the morbidity and mortality rates, by the dissemination methods, and by stability in the environment and capacity of synthetic engineering (Table 1) [2-4].

Table 1. Classification of the Centres for Disease Control and Prevention

\begin{tabular}{llll}
\hline Class of risk & Category $A$ & Category B & Category C \\
\hline $\begin{array}{l}\text { Type of } \\
\text { microorganism }\end{array}$ & $\begin{array}{l}\text { High risk of dissemination, } \\
\text { easy transmission }\end{array}$ & $\begin{array}{l}\text { Moderately dissemination, } \\
\text { lower mortality rate, } \\
\text { generally non-lethal }\end{array}$ & $\begin{array}{l}\text { Newly discovered } \\
\text { (engineered in a laboratory } \\
\text { or genetically altered) }\end{array}$ \\
Example & $\begin{array}{l}\text { Anthrax, smallpox, Ebola, } \\
\text { tularemia }\end{array}$ & $\begin{array}{l}\text { Q fever, ricin toxin, vibrio } \\
\text { cholerae, Salmonella }\end{array}$ & $\begin{array}{l}\text { Nipa virus, Hantavirus, } \\
\text { drug-resistant tuberculosis }\end{array}$ \\
\hline
\end{tabular}


Should we analyze as realistically as possible this threat, we must start by assessing the main advantages and downsides of biological weapon use, from the perspective of a non-state actor interested in this topic:

The advantages of biological weapon use are represented by the relatively accessible and well-known production - cultures on selective or non-selections mediums that can be purchased easily (they may even be found in the laboratories of Medicine or Biology Faculties) - and by the production methodologies like those of yoghurts, beer, antibiotics, or vaccines. Most equipment is also used in the civil industrial environment; thus, it is free to purchase, and control protocols are not active in this field. Dissemination devices are not big; they can be masked easily in crowded areas; small doses of infecting agents are necessary: the rest of the disaster is the product of contagiousness. Mass infection may evolve as an epidemic, which may produce a great number of victims in a noticeably short timeframe, before any defense or public health system may identify the source of the infections as being hostile. Therefore, a specific character of biological weapon use is that it skips the immediate spectacular impact: there are not many victims at once; no explosible is used to destroy important or strategic buildings; the unpredictable character sabotages the defense systems of the states involved, while the greatest hit is not merely the perturbance of the defense system, but also the collective panic created. Moreover, genetic bioengineering and medicine have evolved so much, that we are discussing the existence of superviruses or super-bacteria capable of sabotaging the existing diagnosis and treatments for the standard forms of infections. This aspect was added to the wild virus strains that have caused natural epidemics in the past few years and that are much prone to becoming new biological weapons.

The downsides of using biological agents in a hostile manner are related to the lack of predictability in what concerns the dissemination methods, which must be adapted to the characteristics of the agent in question, to the incubation period, to the distribution inconstancy depending on the pedoclimatic factors of the target-area, the susceptibility and the different response of the human body to infection (immunity, development maturity, associated chronic diseases), space contamination that is impossible to quantify, the possibility of infecting the persons directly involved in the handling, processing, and distribution of pathogenic agents.

For a biological agent to become a biological weapon, a transformation is nonetheless necessary, entailing the existence of logistics and of conditions such as: determining constantly the desired effect - death or disease; having enough minimally infecting doses for a great number of targets; ensuring high infectiousness and contagiousness, predictability, and as short an incubation period as possible; being hard to diagnose and to treat, easy to handle and to store, to reproduce in a synthetic environment; low purchase costs; high natural availability; easy to reproduce; stable outdoors; low persistence after delivery. At the same time, in this scenario, the target population should have low natural immunity for the causing agent and limited or nonexisting access to immunization, diagnosis, or treatment, while the aggressor requires wellestablished means of protection against the infection.

The logistics necessary for the development of a biological weapon may be divided into three categories:

$>$ The means known: they refer to the well-known risk infrastructure (laboratories, companies within the chemical or pharmaceutical industry, toxin development facilities, dissemination means), but also to the infrastructure that may be used; for all of them, there is a possibility to assess the risk and to formulate safety and long-term and short-term intervention protocols.

$>$ The alleged means refer mostly to the threats transmitted without evidence - terrorist organisations - about the investigations concerning the purchase of equipment that may be used in the field, without being able to point out their final purpose, thus to cast suspicion on the states failing to observe The Convention on the Prohibition of Development, Production, and Stockpiling of Bacteriology (Biological) and Toxin 
Weapons and on their Destruction, as it is the case for Russia, China, the US, or the states currently posing a global threat: Afghanistan, Iraq, or North Korea. For instance, in case of smallpox, though officially all virus stocks have been destroyed, their inexistence cannot be guaranteed. Furthermore, whereas the International Geneva Convention has been signed by several states, it has failed to establish any protocol for assessing the nonproliferation measures of biological weapons. The US have opposed such assessments, claiming it would threaten the secrets of the country's industrial bioengineering.

$>$ The unpredictable means, based on modern biotechnologies and on the research in the field of genetic engineering, with the possibility of developing genuine biological hazards, against which there is no safety or protection protocol.

\section{The purchase of biological agents}

The acquisition of pathogenic agents represents the first step in the production of biological weapons. This may be simple or quite difficult, depending on the type of microorganism, on its dissemination and resistance in the natural environment, on the existence of some control regarding the stocks of viruses or bacteria or the available infrastructure.

Biological agents may be purchased as follows:

- With support from a government (states funding terrorism)

- By hijacking the shipments or national programs involving biological agents

- From natural sources (endemic areas where microorganisms have well-known natural reserves)

- By stealing from microbiology laboratories, universities, or research institutes (mostly from those abandoned and not checked, such as those in the territory of the former USSR) [2,3-5].

\section{The production of biological agents}

There are several methods to isolate, purify, and cultivate pathogenic agents, in general; such methods are usually quite inexpensive. In case of toxins, infrastructure and knowledge may be simpler [5,6].

Information concerning the resistance of microorganisms, the required culture mediums, details on infections may be found on the Internet, in libraries, in manuals of biology genetics, infectious diseases, or microbiology.

In order to produce biological agents, you need the basic materials and two other things:

- A laboratory: an improvised laboratory may be set up for less than USD 10,000; one may choose the so-called double-use devices - old equipment from drug industry, fermentation devices from dairy industry, or a simple centrifuge - that are slightly altered.

- Specialists: usually, this requires mid-level or high-level training (biologists, chemists, geneticists). Many members of the terrorist groups were professionally trained in the European or American states (e.g., the pilot having hijacked the plane towards World Trade Centre had studied electronics in Germany, France, Russia, and the UK). Even in Romania, dozens of students from the Arab countries have trained in the fields of biology and chemistry. Another example raising suspicions refers to the former specialists within the Russian network BioPreparat - accounting for over 32,000; following the restructuring and destruction of the research facilities in their country of origin, they have migrated - coincidentally - in states developing biotechnological research programs.

The production of biological agents ends with the final dissemination materials, providing a generous environment and stability when handling and containing the microorganism until dispersion. These materials are usually liquid suspensions or powders [6-9]. 


\section{Transportation of biological agents}

The arming devices of biological agents do not involve any advanced technique; the solutions or powders with biological contents can be transported in the most usual items, such as pen caps, letters, or packages with non-dangerous contents, in powders that look like spices or in liquids that look like cleaning products or like beverages. Virtually any item can be transformed into a transportation device, mainly because - on a general note - the amounts necessary for the success of an attack are not related to quantity, but to the dispersion method and to the infectiousness level, which will occur automatically once the plan is implemented. Another advantage of transporting biological substances is the lack of protocols or assessment standards meant to identify the presence of a dangerous material (such as detection systems for drugs or nuclear materials).

\section{Dissemination of pathogenic agents}

The dispersion of biological agents is the key for producing a massive biological attack. Whereas of a great importance, as in the case of transportation, dispersion does not require advanced technologies, but it uses the existing technique within the urban infrastructure:

$\checkmark$ For the aerosols, exposure through inhalation can be achieved by sending trap letters or packages, agricultural powders, fire hydrants, pharmaceutical inhalers, deodorisers for the inside and for the outside, and mostly through the ventilation systems; in the open space, all it takes is to simply open a flask or use a pressure device, given that dissemination is ensured by air currents.

$\checkmark$ The contamination of the water sources, of the sewer and drinking water sources, even of the water sources for the sprinklers used in parks or botanical gardens.

$\checkmark$ The contamination of basic consumption products at the production sources or of foods for restaurants and consumption networks (cafeterias, bars, food-courts).

$\checkmark$ The contamination of products provided in the medical system (needles, syringes, surgical devices)

$\checkmark$ Military projectiles or ammunitions.

$\checkmark$ Indirect transmission through vectors (fleas, mosquitoes, rats).

$\checkmark$ The contamination in agricultural environment - contaminating cultures or making the livestock sick or even contaminating the slaughterhouses.

By far, the preferred form of dispersion is the one of inhalation in open space; in order for this scenario to work, several conditions must be met: average heat and humidity, slight wind (5$10 \mathrm{~km} / \mathrm{h}$ ), even landform, circulation of vehicles or of the population, active air conditioning systems, as crowded spaces as possible.

The best advantages of dispersion are represented by the lack of suspicion concerning the persons involved or the illicit procedures and the incubation period of pathogenic agents which until the evolution of the disease and the identification of the attack - provide a time window allowing the perpetrators to run by covering rather important distances.

\section{Paths of exposure to biological agents}

a. The respiratory path

Most chemical and biological agents have a lower or higher effect on the lungs, even though the primary exposure route was not at that level. The body is the most sensitive and receptive through this path due to the great surface and to the pulmonary gaseous exchange, to the susceptibility of the mucosae to infections and to phagocytes. The last - if they do not manage to destroy the pathogen - will carry it into the lymphatic system and then into the blood stream. Furthermore, through inhalation, the onset of the infection occurs much more rapidly than in case of digestive or epidermal exposure.

b.Skin and mucosae exposure 
Unlike chemical agents, easily absorbable through the skin, biological agents fail to penetrate the cutaneous barrier, except for the case where it features lesions such as wounds, irritations, or burns. Thin and highly vascularised skin areas are the most prone to catching pathogenic agents, just like hot and moist environment, leading to pre-dilation, which increases receptivity for microorganisms. Mucosae are significantly more likely to get infections in case of both dispersion paths; the clinical onset of the disease is much delayed compared to the other potential dissemination paths. It often starts with lesions at the level of the inoculation areas. The epidermal path is not an election path, given that exposure may be pathological only for a small part of the target-population. For high receptivity, very irritating substances are required, able to break down the cutaneous barrier.

c. The digestive path

This simple path has been used previously in several attacks through the contamination of the water sources and of the food, by taking the hand to the mouth after touching contaminated surfaces or by swallowing the respiratory mucus following inhaling exposure. This scenario is simpler to assess through a rigorous control of foods and beverages commercialised and through strict sanitary measures in the food industry. The public health and epidemiology systems conduct such checks in most states of the world, but there are suspicions related to sabotage or to contamination plans right after the checks. In this case, too, the incubation periods are generally longer than in case of respiratory contact, but symptomatology can be as severe, given the risk of systemic impairment.

d.An equally realistic scenario is the one where the primary attack targets the agrarian cultures or the livestock, with the purpose of causing economic loss, the destruction of crops, and the secondary sickness of the population exposed; the same plausible scenario includes the possibility of suicidal bioterrorism: namely, a suicidal attacker - ready to become exposed to a contagious infection, meant to spread to the communities visited. As it may be noticed, no supposition is too far-fetched when man's fear of uncontrollable phenomena exceeds the limits of the possible.

\section{Specific risk analysis}

\section{Identification of risk factors}

Risk analysis represents the foundation for developing biosecurity defence and intervention protocols. The first step of the analysis is to define the nature and purpose of the threatening phenomenon. Furthermore, it is necessary to get a better insight into the determining factors and the characteristics deriving from these notions:

- Firstly, it is best to avoid political or academic contentious debates in what concerns the nature of terrorism and to focus on the essential matter, namely, biological weapon use by nonstate actors. On principle, the notion of non-state actors refers to organisations completely independent from government funding and control, usually pertaining to or developed by the civil society, operating in transborder networks, with the main purpose of producing various consequences in the states where they carry out their activities. Consequently, we will refer to non-state actors as organised crime networks, insurgents, or terrorist cells with no political or military control, acting according to their own laws, religions, motivations, with the purpose of inducing a state of terror in the society: thus, the primary target is the psychological impact of the action.

- Biological weapon use by non-state actors does not necessarily aim mass murder. Equally realistic, there is the desire to incapacitate the target population temporarily in order to influence the social and political will or to contaminate a certain space, thus creating economic damage and social imbalances. 
- Thirdly, it is worth highlighting that the human species is not the only target of bioterrorism. Agricultural structures - livestock, cultures - can be equally destroyed during an attack, with the purpose of producing material losses or as a source of contamination for people.

There are several characteristics of bioterrorism to take into account in analysing the contents of this phenomenon:

- The raw material of the biological weapon: bacteria, viruses, or toxins (they do not multiply and they are not contagious; hence, they are similar to chemical weapons).

- A biological agent is not the same as a biological weapon. Whereas very dangerous for health, a biological agent becomes a weapon only after a sufficient amount of it is introduced into a specially designed or an artisanal dissemination system, having the capacity of being dispersed on significant distances, hence targeting as many people as possible.

- The relatively substantial incubation period for any biological weapon is a double-edged sword, both edges aiming the target-entity: on one hand, it provides the perpetrators with enough time to leave the crime scene; on the other, it delays forensic investigations and medical intervention.

- In what concerns the contagious biological agents, the attack involves secondary, tertiary impairment, etc - on a considerable distance in time and space - of the populations beyond the one targeted initially. Such consequences may be difficult to control even with the most modern public health systems and they represent an even greater danger than the nuclear weapons.

- There is a substantial difference between the development of biological weapons by military scientific organisations (devices adapted to the culture, reproduction, and even dissemination depending on the pedoclimatic conditions within the target-area) and their use by non-state organisations, aiming to stir chaos through attacks using artisanal, primitive devices and agents.

- Prophylaxis and the development of a good defence represent the difference between a mass massacre and a couple of victims. Th drills conducted in the USA have proven that an effective coordination of the intervention, a knowledge of the protocols, and early medical measures lead to a decrease in the number of possible victims of a biological attack from 120,000 to 35,000 in case of very serious infections.

- However, many other variables are still not quantifiable: new technologies, modified bacteria and viruses, ever more original dissemination methods. For instance, before the "Anthrax letters" in 2001, nobody had taken into consideration the possibility of this dissemination method.

\section{Analysis of the reality of bioterrorist danger}

The main fundament of the risk analysis is the fact that the insufficient evaluation of the risk for such a phenomenon leads - beyond doubt - to a sub-optimal management of such an event.

The first principle of calculating biological hazard is that the essential threat is not represented by the epidemiological and medical of the biological agent, but by the capacity of certain people or organisations of using the agent in question with hostile purposes. Practically, even the most dangerous microorganism represents a very low risk for the population, as long as nobody is willing to use it as a mass destruction weapon. Reversely, even the most diabolical hostile intentions remain worthless if the subject fails to provide and use the agent in question with the aforementioned purpose.

Following this idea, we present a first equation for calculating the biological risk, using the terms below:

- BAIR - bioterrorist attack incidence risk;

- CA - consequences of the attack;

- PA - probability of the attack

$$
B A I R=C A * P A
$$


Each element of this equation may be further developed as follows:

CONSEQUENCES OF THE ATTACK $=$ Value of the target to protect $\times$ Pathogenic
potential of the biological agent $x$ Vulnerability of the target to protect

PROBABILITY OF THE ATTACK = Perpetrator's motivation for using a biological weapon X Perpetrator's capacity of getting such weapons $\quad[3,7]$

\section{Value of the target to protect.}

The main value of the target to protect in case of bioterrorism is represented by the overwhelming number of endangered human lives. This value actually provides the essence of biosecurity and - in order to defend life - no risk or cost is too high.

\section{Pathogenic potential of the biological agent}

The analysis of the potential pathogen of biological agents is delimited to the medical field, more precisely to the public health specialists, to epidemiology, infectious diseases, emergency medicine, laboratory medicine, pathological anatomy, and forensic medicine. The analysis consists in the evaluation of the characteristics specific to each pathogenic agent of risk, and if analysis is nonspecific, it will be necessary to underline their general characteristics (species characteristics; infectiousness groups; treatment groups; known, unknown, or newgeneration agents). The potential of the biological agent does not refer only to the medical impact, but also to the psychological impact that such an event has upon the level of the targetcommunity.

\section{Vulnerabilities of the target to protect.}

The main vulnerability is represented in this case by the sensitivity of the population to the infection with the pathogenic agents of risk. The analysis of the vulnerability in case of bioterrorism involves the identification of the paths through which the target - population, city, strategic building - can be attacked by non-state actors (food supply system, the educational facilities system within a city, the hospital system, the positioning of strategic buildings, the crowded or tourist areas, etc).

\section{Capacity of the organised groups of obtaining biological weapons and of initiating a biological attack.}

In what concerns conventional terrorism, the discussion focuses on factors such as the training of terrorists, the funding of the group, and the communications that they use. In the context of bioterrorism, the analysis should focus on the biological weapons and on their purchase and use. As for the purchase of biological weapons, many analysts posit that the old obstacles in their acquisition and development have eroded so much, that it may well be assumed to be very easy for certain terrorist groups. An important aspect of this analysis is that no terrorist - however insignificant his present activity may be - is incapable of using biological weapons in the future; non-state actors are adaptable, even innovative persons when it comes to attaining their goals and to following their own motivations fanatically; such motivations - no matter how obscure and archaic in the eyes of modern society - should not make us believe that they are not aware of the latest technological novelties. Scientific and technological evolution, as well as the progress of communications, transportation, and commerce open new perspectives for terrorists, thus leading to new methods of attack.

The calculation of terrorists' capacity to provoke biological attacks analyses a series of factors, namely:

The organisational capabilities: the technical and tactical requirements for the production of biological weapons involve a network-based organisation with numerous members, with well- 
defined structures and with a central management ensuring leadership of all subordinated departments.

The financial resources: an essential condition for the production of biological weapons is the level of funds managed by that group in order to purchase the equipment and materials necessary for practices, for building facilities, and for paying the staff involved in the project.

The logistical resources: besides the financial and organisational qualities, a criminal group also needs logistic support (i.e., proper transportation and communication means and operational bases); it is even more important for this type of logistics to be difficult to track and follow. Another essential condition of criminal logistics is the existence of a well-controlled transborder network, meant to ensure the purchase of basic materials and equipment, as well as their shipment to the destination. Unfortunately, this phenomenon is hard to trace because many such pieces of equipment have double or even triple use and the checks concerning international trade are not well implemented in this respect. Biological materials may be hidden in almost any type of usual items serving as containers; there is no standardised control for their detection at the border points or in airports.

The purchase of necessary knowledge and skills may be ensured in two ways: the development of existing knowledge within the group or their acquisition from persons outside the group. Regardless of the manner of acquiring the necessary knowledge, technical and scientific learning involves explicit acquisition of notions (manuals and scientific treaties) and tacit acquisition, through failed or successful experiments and through practice. There is a major difference between theoretical and practical knowledge regarding the development of biological weapons; however, in the world of terrorists, any person without experience can transcend an ascending study curve. In the recent years, almost no form of education is reserved only to the traditional education system. The specialised treaties, books, and papers available online provide the possibility of self-education, but no tacit knowledge. On the contrary, globalisation has provided access to studies in the field even to future criminals and terrorists who migrated as students to all renowned universities around the globe; in its turn, this provided access to both information and practice. A more viable option - directly related to the material funds of the organisation - would be to hire specialists; we refer here to the former employees of the biotechnological military research bases who worked in biological weapon development programs, with government support (the former programs of the Soviet Union - after the official rescinding of the laboratories, many researchers left Russia; the South-African programs; and Iraq). It is assumed that a skilled microbiologist and an engineer designing the dispersion method would be enough to create a biological weapon.

The purchase of necessary materials and technology. The material necessities for a biological weapon include a sufficient stock of pathogens, specific culture mediums ensuring an easy growth of microorganisms, and fermentation/lyophilisation equipment. Production success generally depends on the production scale and on the type of organism produced. Commercial or standard lab pieces of equipment may suffice for the small-scale production of agents. On the contrary, the creation of large amounts and the application of special processes - such as microencapsulation - require significantly more complex endowments.

The actual production of the biological weapon. Once purchased, pathogens need growth and reproduction in high amounts. The simple culture of microorganisms is not enough for producing biological weapons. They must be cultivated in such a manner as to preserve every percentage of the virulence and they must be stored in safe mediums, ensuring their stability and survival until the next step of biological weapon design. This is also the step during which one may alter the resistance and virulence structures modifying the activity of the biological agent. The most important point worth noting in this stage is the safety of persons handling the agent; the risk of infection is rather high among them. For an experienced microbiologist, these aspects do not represent a problem at all; whereas, thus far, modified bacteria have been considered inaccessible for terrorists, this idea is no longer of actuality, given that the former Soviet programs 
along with the evolution of biotechnology and genetics have long stopped being a strategic secret, while the experience of the former Soviet researchers can be made available to the criminal groups in the current context.

The creation of arming devices and the dissemination of biological agents. Whereas it is true that a few kilograms of biological agent - well purified and filtered, armed accordingly can kill the population of an entire city. However, the truly serious elements are found in the details. The final effect of the attack depends on the extent to which the biological agent was armed. The terrorists' method of choice is the one of particles suspended in aerosols, as spray or powder. The method has proven very effective, though the technology of aerosolising the agent is quite difficult, and the presentation form has several downsides: oxidation, sunlight, humidity, and other environmental factors can reactivate a series of agents before reaching their targets. The other dissemination forms (the contamination of water, of food; a terrorist getting infected in order to spread it to others by direct contact) are also as realistic as possible as scenarios in risk analysis, but the main problem remains the obvious preference for the method of aerosols and for the methods of dissemination not requiring the special design of a device, but the use of existing devices (dispersion from a plane; contamination of ventilation systems, or short-circuiting of air filters; artisanal devices for crowded open spaces, etc).

The issue of government sponsorships: some states are known to shelter, support, handle, and direct the networks of insurgents or other non-state violent organisations, in order to impose their own policies beyond the frontiers of the state or at domestic level. The same states providing moral, logistic, and material support to terrorist organisations are those known to have used biological weapons. There are two possibilities in this respect: the state offers support to the terrorist network in order to create unconventional weapons or the state provides terrorists with a biological weapon already perfected and functional, developed through paramilitary research projects. The latter is analytically improbable because - however strong the ideological connection between the government and the groups in question - it is not considered realistic for such a complex weapon to be left in the hands of totally inexperienced persons in the field. Nonetheless, should the government of a state wish to attack another state without having the source determined, the most convenient scenario is to mask the offensive endeavour as terrorist intentions and motivations.

\section{The terrorists' motivation of using biological weapons.}

For the likelihood of a biological attack to be significant, both the skills of certain groups in using the biological weapons and the motivation of leading the war in such a manner are required. The analysis of motivation is supported by ideological, strategic, and tactical factors each with its stimulants and constraints - all together determining the terrorists to act or not at this level.

Ideological motivations represent the political, religious, sociocultural factors categorising specifically the beliefs shared by the members of a group. The analysis of this factor determines whether a terrorist network may consider biological weapon use as being tailored to its ideology and its purpose. For instance, extremist groups with religious-apocalyptical views may see biological agents as the divine tool for bringing the end of the world; similarly, racist groups may consider that - through infections with certain pathogens - they can target only one ethnic group within the population. Other groups posit that the "reputation" conferred by biological weapons or by mass destruction weapons is welcome and sufficient for their purpose and that it provides more credibility to them, more status and value in the media, which has a strong influence on public opinion [2,8-9].

Strategic motivations are the specific factors determining the purpose of an organisation - political, religious, etc - and they refer to the intention and the means for attaining the purpose in question. Biological weapon use may have several specific outcomes, with a special 
importance for terrorists: significant number of victims; economic damage; contamination of the target-area; interruption of strategic functions (transportations, water and food supply) and undermining of the authorities by destroying public trust. Furthermore, the simple threat with a biological attack serves as a very effective blackmail tool [10,11].

Tactical motivations refer to the means and techniques through which the terrorists can attain their objectives. In the analysis of this factor, we take into account all the advantages and the downsides of biological weapon use, supported by the ideological and strategic foundation of the entire potential.

The analysis of the motivation and of the capacities involves tools that identify and examine general and specific behavioural patterns of certain terrorist groups, upon evaluating all the factors involved. These behavioural tools - the ideology, the analysis of past attacks, the interorganisational dynamism, the perception of risks, etc - are prognosis indicators for certain types of attacks that may be used by terrorists.

\section{Prevention against bioterrorism and biological warfare}

Prevention involves multiple measures to develop a safety network against the use of mass destruction weapons. First of all, it is necessary to understand the phenomenon with all its meanings: comprehensively (all the aspects of the phenomenon are important and they play a role in risk analysis, not only the pathogenicity of biological agents), realistically (becoming aware of the risks beyond the sombre patterns of the diseases caused and the great number of victims - the insufficient information leads to eluding important aspects), and flexibly (the development of the new genetically modified agents, the emergence of new viruses involves a more comprehensive view of the concept, in order for it to adapt constantly to the different nature of the risks) [7-9].

Another important objective of prevention is to consolidate the global standards of biological weapons. Human life is obviously superior to any racial, religious, social, cultural, or political standards; hence, beyond the differences in opinion and the tensions between the governments of certain states, it is essential to implement an international cooperation meant to reach a unitary view of the anti-bioterrorist concepts. These global standards refer mainly to the development, consolidation, and standardisation of biosafety efforts. The topic of these efforts is represented by the sources of biological agents, which can be hospital laboratories, university laboratories, research institutes, etc. All of them are prone to hijacking, due to the weak security procedures. Two measures can be taken by the international community, in order to remove the exposure risk:

- Creating a list of high-risk biological agents to be internationally validated and

unanimously accepted. These lists and rankings exist in some states and they are notified by WHO, but there is no unique international validation. This list should be updated regularly and adapted to the new medical, biotechnological, and genetic discoveries. Such a concern is on the agenda of the Australia Group and of the Convention for Biological Weapons - two entities conducting their activity regularly and the endeavours of which aim to implement biosecurity, but which are not recognised at the same level internationally.

- Establishing an international convention regulating legally and politically the obligation of transferring and handling biological agents in a secure manner. A specific proposal on this topic is the development of recording, identifying, and monitoring systems of hazardous biological agents, the determination of a standardised method of "inventorying" them, the imposition of strict export and import standards for the recorded agents, as well as support and assistance procedures for the Member-States for implementing the measures imposed [3,10-12].

The improvement of the collaboration between defence systems, law enforcement bodies, and the field of science is another important attribute of prevention. Whereas, in case of conventional terrorism, it addresses the legal, forensic, domestic, and foreign or military services, the first defence line of bioterrorism is the public health system; hence, all defence protocols in the field of bioterrorism are based first and foremost on prophylaxis, then on rapid and effective treatment, which means that all governmental structures require epidemiological and medical 
expertise in order to build a valid, logical, and strong safety network, dealing with both the terrorist phenomenon and the biological threat. A vulnerability in the biosecurity network is represented by the fact that many developing countries are actually not medically prepared to face a bioterrorist threat [8-14].

The international uniformization and implementation of medical prophylaxis and intervention guides, as well as the possibility of assistance from foreign programs in case of an attack represent the most effective prerogative of biosecurity improvement. Regular and effective risk analyses should be made available internationally, for all the countries with deficits in the fields of defence to be able to implement nationally the knowledge acquired, depending on their own vulnerabilities and advantages.

Another important aspect is the communication protocol with the population. In case of the possible attack, the general response is dominated by panic and reflex reactions of dread, despair, leading to an inadequate and inefficient behaviour that ends up blocking the medical system, first of all. We can avoid this problem by getting the population ready, which is achievable through information and constant education. Furthermore, guidelines may be developed for the general population, ensuring that in a risk situation, people still trust the defence mechanisms; this will lead to a calm behaviour in such situations. The main protection lines of the population against fear are the efficient handling of panic, the minimisation of rumours, hearsay, apocalyptical scenarios, as well as media news that often paint the real situation in darker tones, given the informational competition [12-14].

Not least, international cooperation concerning the systems of investigations, information, and law enforcement - for constant and updated information exchanges in what concerns illegal, terrorist, or highly suspicious activities - is essential for the primary prevention of biological risk.

\section{Conclusions}

The topic of biological weapons may be discussed in the context of both terrorism and power games at the level of the main states because biological attack in a war - even in a theoretical one - would represent the guarantee of a victory with minimum infrastructure and logistics and with minimum human life losses on the side of the attacker. As improbable as it may seem at first glance, in such a scenario it is important to prepare an effective defence, more given the international actuality, so much stricken by the terrorist phenomenon.

Collaboration represents the keyword in the development of defence mechanisms against bioterrorism; this relationship should be implemented both nationally and internationally and it should comprise all competent bodies in the fields correlated with this phenomenon, given that all of them are involved in both prevention and short- and long-term response mechanisms.

\section{References}

[1] Romanian Service of Information. Bioterrorism and biological weapon. https://intelligence.sri.ro/bioterorismul-inamicul-invizibil/

[2] L.H. Kahn, Biodefense research: can secrecy and safety coexist, Biosecurity and bioterrorism: biodefense strategy, practice and science, 2, 2004, pp. 81-95.

[3] E.J. Suk, A. Zmorzynska, I. Hunger, W. Biederbick, J. Sasse, H. Maidhof, J.C. Samenza, Dual-use research and technological diffusion: reconsidering the bioterrorism threat spectrum, PLoS Pathog., 7(1), 2011, pp. e1001253.

[4] A. Tegnell, F. Van Look, A. Baka, S. Wallyn, J. Hendriks, A. Werner, G. Gouvras, Biological weapons - development of a matrix to evaluate the threat of biological agents used for bioterrorism, Cell. Mol. Life Sci., 63(19), 2006, pp. 2223-2228.

[5] L. Allen, Biosecurity and Non-communicate Diseases, J. Bioterrorism Biodefense, 7(2), 2016, pp. 1-4. 
[6] A. Anghel, V. Dulgheru, Terorismul actual, risc major la adresa securitatii commune a Europei, Rev. Stiinte Militare, 2, 2017, pp.53-58.

[7] V. Barras, G. Greub, History of biological warfare and bioterrorism, Clin. Microbiol. Infect., 20(6), 2014, pp. 497-502.

[8] B. Bodowle, S.E. Schutzer, A. Einseln, L.C. Kelley, A.C. Walsh, J.A. Smith, B.L. Marronne, J. Robertson, J. Campos, Public health, building microbial forensics as a response to bioterrorism, Science, 301(5641), 2003, pp. 1852-1853.

[9] O. Cenciarelli, V. Gabbarini, S. Pietropaoli, A. Malizia, A. Tamburini, G.M. Ludovici, M. Carestia, D. Di Giovanni, A. Sassolini, L. Palombi, C. Belleci, P. Gaudio, Viral bioterrorism: learning the lesson of Ebola virus in West Africa 2013-2015, Virus Resp., 210, 2015, pp. 318-326.

[10] C.A. Cummings, D.A. Relman, Genomics and microbiology, microbial forensics - "crossexamining pathogens", Science, 296(5575), 2002, pp. 1976-1979.

[11] T. Balzacq. The three faces of securitization: political, agency, audience and context, Europ. J. Int. Rel., 11(2), 2005, pp. 173.

[12] P.K. Dash, M.M. Parida, P. Saxena, M. Kumar, A. Rai, S.T. Pasha, A.M. Jana, Emergence and continued circulation of dengue-2 (genotype IV) virus strains in northern India, J. Med. Virol., 74(2), 2004, pp. 314-322.

[13] J.B. Petro, T.R. Plasse, J.A. McNulty, Biotechnology: impact on biological warfare and biodefense, Biosecurity and bioterrorism: biodefense strategy, practice and science, 1(3), 2004.

[14] B. Fischhoff, R.M. Gonzalez, D.A. Small, J.S. Lerner, Evaluating the success of terror risk communications, Biosecurity and bioterrorism: biodefense strategy, practice and science, 1(4), 2004, pp. 255.

Received: September 30, 2020

Accepted: February 2, 2021 